largest increases in CRS over time. Patients aged $\geq 70$ years had the highest probability of death in the first year after diagnosis $(34.9 \%)$, but the conditional probability of death in the $2 \mathrm{nd}, 3 \mathrm{rd}, 4 \mathrm{th}$, and 5 th year declined abruptly to $14.7 \%$, 9.2\%, 6.0\%, and 4.9\%, respectively.

Conclusion The CRS rates for patients with ovarian cancer improved over time, particularly among patients with poorer initial prognoses. Our estimates can enable patients to make better informed decisions regarding follow-up care and their personal life.

Disclosures I have no conflicts of interest.

\section{EVALUATION OF SURVIVAL OUTCOMES FROM DELAYED CYTOREDUCTION SURGERY FOLLOWING NEOADJUVANT CHEMOTHERAPY IN ADVANCED EPITHELIAL OVARIAN CANCER}

${ }^{1}$ Shih-Ern Yao, ${ }^{2}$ James Nicklin. 'Queensland Centre for Gynaecological Cancer; Level 6, Ned Hanlon Building; Royal Brisbane and Women's Hospital; ${ }^{2}$ Queensland Centre for Gynaecological Cancer

\subsection{6/ijgc-2020-ESG0.101}

Introduction/Background Optimal timing of cytoreductive surgery following neoadjuvant chemotherapy (NACT) has not been established in the treatment paradigm of advanced epithelial ovarian (EOC) cancer. Traditionally, interval cytoreduction surgery (ICS) is undertaken following 3 cycles of treatment, however in a proportion of patients, surgery is delayed for reasons including incomplete disease response, poor surgical candidacy and anticipated suboptimal tumour resectability.

We looked to investigate survival outcomes in advanced epithelial ovarian cancer (EOC) patients with the intention of maximal cytoreduction following neoadjuvant chemotherapy (NACT) with respect to timing of surgery and degree of cytoreduction.

Methodology A retrospective review was conducted of 572 patients with EOC treated with NACT with the intention of interval cytoreduction surgery (ICS) between 2008 and 2017. Overall survival (OS) and progression-free survival (PFS) outcomes were analysed and compared with patients who only received chemotherapy. Outcome measures were correlated with the number of NACT cycles and amount of residual disease following surgery.

Results There was no difference in the proportion of patients in whom complete cytoreduction was achieved based on number of cycles of NACT. Median 5-year OS and PFS for patients undergoing cytoreduction after NACT was 38 and 24 months respectively with no significant difference in OS between standard and delayed timing of surgery. Significant OS advantage was associated with patients who had undergone complete cytoreduction compared with those with any macroscopic residual disease $(<1 \mathrm{~cm}$ residual: $\mathrm{HR} 1.68 ; \geq 1$ cm residual: HR 2.77).

Conclusion From this study, survival outcomes do not appear to be worse for patients with EOC treated with NACT if cytoreduction surgery is delayed beyond three cycles. In EOC patients, the imperative to achieve complete surgical cytoreduction remains gold standard, irrespective of surgical timing, for best survival benefit.
Disclosures This work was supported by a research grant from Gynaecological Cancer Research Education and Development Society.

Neither author disclose any conflict of interest

\section{PREOPERATIVE EVALUATION OF LIPID MARKERS OF MALIGNANT EPITHELIAL OVARIAN TUMORS}

${ }^{1}$ Mariia lurova, ${ }^{1}$ Stanislav Pavlovich, ${ }^{2}$ Nataliia Starodubtseva, ${ }^{2}$ Vitaliy Chagovets, ${ }^{2}$ Grigorii Khabas, ${ }^{2}$ Vladimir Frankevich. ${ }^{1}$ Federal State Autonomous Educational Institution of Higher Education I.M. Sechenov First Moscow State Medical University of the Ministry of Health of the Russian Federation (Sechenov University); Federal State Budget Institution 'national Medical Research Center for Obstetrics, Gynecology and Perinatology Named after Academician V.I. Kulakov' Ministry of Health of Russia; ${ }^{2}$ Federal State Budget Institution 'National Medical Research Center for Obstetrics, Gynecology and Perinatology named after Academician V.I. Kulakov' Ministry of Health of Russia, Moscow, Russian Federation

\subsection{6/ijgc-2020-ESG0.102}

Introduction/Background The venous blood is repleted with abundant tumor-promoting factors and lipids, that play an essential role in ovarian high-grade serous carcinoma (HGSC). A comprehensive picture of mediators impacting HGSC progression is, however, not available.

Research question to determine the value of the serum lipid profile in HGSC for diagnosis.

Methodology This study was approved by the Institute Research Medical Ethics Committee. Analysis of blood serum lipids of healthy volunteers ( $\mathrm{n}=13$, control group) and patients with verified HGSOC (I-IV stages, $\mathrm{n}=28$, main group): I-II stages $(n=5)$, III-IV stages $(n=23))$ has been performed. Patients with HGSOC managed in the Department of Innovative Oncology and Gynecology (National Medical Research Center for Obstetrics, Gynecology and Perinatology named after Academician V.I. Kulakov) were comparable in age, body mass index, grade and FIGO stages. Lipids were analysed by high performance mass spectrometry liquid chromatography (HPLC-MS). The Orthogonal Projections to Latent Structures Discriminant Analysis (OPLS-DA) multifactor analysis method and non-parametric t-test, have been applied for statistical data processing. Random forest model was used to evaluate predictive performance of potential biomarkers based on leave-one-out crossvalidation in terms of area under the receiver operating characteristic (ROC). The predictive accuracy of the predictive lipids was performed using the logistic regression modeling with AUC value.

Results In main group the levels of 128 of 345 studied lipids differed significantly compared to the control group $(\mathrm{p} \leq 0.05)$, the parameters of the OPLS-DA model were: $\mathrm{R} 2=$ $0.87, \mathrm{Q} 2=0.80 ; \mathrm{AUC}=0.99$. ROC curve sensitivity $=96 \%$ and specificity $=1 \%$, the AUC value of these metabolite combinations for predicting HGOC recurrence was 1. Lipid profile changes significantly differed between the groups: control group vs I-II stages $(\mathrm{p} \leq 0.05)$, control group vs III-IV stages $(\mathrm{p} \leq 0.05)$.

11 patients who developed the disease relapse or progression had significant preoperative increase of oxidized lysophosphatidylcholine (OxLPC) and phosphatidylethanolamine (PE) in contrast to 17 patients who showed no evidence of recurrence after at least 14 months of follow up. 